\title{
Filter-based Control for Parallel Plate Micro Electrostatic Actuators
}

\author{
Mohammad H. Salah \\ Department of Mechatronics Engineering \\ Hashemite University \\ Zarqa 13115, Jordan \\ msalah@hu.edu.jo
}

\author{
Alper Bayrak and Enver Tatlicioglu \\ Department of Electrical and Electronics Engineering \\ Izmir Institute of Technology \\ Urla, Izmir 35430, Turkey \\ \{alperbayrak, envertatlicioglu\}@iyte.edu.tr
}

\begin{abstract}
In this paper, a filter-based nonlinear control strategy for parallel-plate micro electrostatic actuators is designed. The proposed control technique utilizes the measurements of the micro actuator's movable plate displacement and the device internal charge. The information of the micro actuator's movable plate velocity is utilized as well in the control synthesis but since it is difficult to be measured, filtered signals are designed and utilized to facilitate the control development. A Lyapunov-based analysis is presented which proves that a desired time-varying displacement of the micro actuator's movable plate is accurately tracked. The proposed nonlinear controller is capable of controlling the movable plate beyond the pull-in boundary that is one third of the capacitive gap. Representative numerical simulations are introduced to demonstrate the performance of the proposed filter-based nonlinear control strategy in accurately tracking the deflection of the micro electrostatic movable plate within the entire capacitive gap. Finally, a comparison with a standard PID controller is also presented to demonstrate the effectiveness of the proposed control design.
\end{abstract}

Keywords-nonlinear control; MEMS; electrostatic actuator

\section{INTRODUCTION}

Micro electrostatic actuators (MEAs) are widely employed in Micro Electro Mechanical Systems (MEMS) applications due to simplicity of structure, ease of fabrication, and possibility of quantification of micro-scale electrostatic forces. Electrostatic actuators are used widely in MEMS such as micro-mirrors, optical gratings, variable capacitors, accelerometers, precise positioning, large vertical displacement, fatigue testing of low-stress thin films, blood vessel manipulation, and optical scanners.

The region of stable and controllable motion for MEA is limited by a bifurcation phenomenon called "snap-through" or "pull-in" and that is one third of the micro actuator capacitive gap. Pull-in can happen for voltages lower than the static pullin value, depending on the initial state variable values and external applied forces [11]. The main challenge is to extend the travel range of the parallel plate micro electrostatic actuator beyond the pull-in limit that is one third of its full capacitive gap. The region of attraction of the equilibrium point may be quite small, particularly near the bifurcation point. It is required to find a voltage control law that can be used to stabilize any equilibrium point in the gap and to provide a large region of attraction.

Seeger and Crary [14] presented a simple method to stabilize the micro actuator by adding a series capacitance. This work was extended in [12] with a switched-capacitor circuit to stabilize the device against voltage pull-in provided that the parallel parasitic capacitance is sufficiently small. $\mathrm{Lu}$ and Fedder [6] used a pre-filter in front of the feedback loop to shape the input command. The authors of [10] presented a solution to extend the working range of parallel-plate electrostatic actuators by employing a bumper structure in the design of two-beam to change the spring constant near the critical point of pull-in. However, It has been proven that instability is avoided by a sufficient reduction in the drive voltage to achieve full gap operation [11] and that was also verified when Bermejo and Castaner [1] studied the potential use of a photovoltaic source as direct drive of the micro electrostatic actuator. The authors of [2] and [9] demonstrated that electrostatic actuators can be driven based on pulsedcurrent drive that requires much less voltage than voltage drive. In fact, operation of electrostatically actuated MEMS with amplitudes beyond the pull-in instability limit can be achieved with appropriate selection of actuation voltages [4].

Many control strategies have been developed to solve the pull-in instability problem of micro electrostatic actuators. Seeger and Boser [13] presented a circuit that controls the amount of charge on a parallel-plate electrostatic actuator. They showed that charge control increased the stable ranges of motion but the maximum stable deflection is limited due to parasitic capacitance and tip-in. In [7], the authors presented a linear time-invariant voltage controller to extend the travel range. They were succeeded to extend the regions of operation up to $60 \%$ of the initial gap. Zhu et al. [18] demonstrated that traveling range of MEA can be extended to the full gap utilizing charge control and capacitive feedback. In [19, 20], Zhu et al. presented a flatness-based controller to extend the operational range of the MEA to the full capacitive gap. They were able to reduce the air gap to one third of its current dimension using this controller, and that decreased the maximum value of driving voltage. Later on, Zhu et al. [21, 22] presented two robust control laws for a parallel-plate electrostatic microactuator in the presence of parasitics and 
parametric uncertainties. Their proposed controllers were based on input-to-state stabilization and robust backstepping and demonstrated satisfactory and robust performance in numerical simulation. The authors of [16] linearized the micro electrostatic actuator model at multiple operating points and then designed a robust PID control switching scheme for setpoint regulation. Li and Liu [5] proposed a novel adaptive robust tracking control scheme for uncertain electrostatic micro-actuator. The control scheme was based on dynamic surface control method and H-infinity control approach. Finally, Tee et al. [15] presented adaptive control for electrostatic microactuators with bidirectional drive. They showed that the movable electrode is capable of tracking a reference trajectory within the air gap without knowledge of the plant parameters.

In this paper, a filter-based nonlinear controller is proposed for parallel-plate micro electrostatic actuators. The measurements of displacement and internal charge of the micro actuator are employed in the control algorithm to accurately track a desired time-varying displacement of the movable plate. Filtered signals are designed and utilized to facilitate control development due to the lack of information about the movable plate's velocity while other researchers usually utilize reduced-order observer to estimate the velocity such as [8]. A Lyapunov-based stability analysis is utilized to develop the nonlinear control strategy to operate the micro actuator beyond the pull-in instability limit. Representative numerical results are presented which demonstrate the performance of the proposed control approach.

This paper is organized as follows. In Section II, the micro electrostatic actuator dynamic model is presented. In Section III, the nonlinear controller is formulated and proposed. Section IV presents the filter design to facilitate the control development in the absence of movable plate's velocity measurement. In Section V, numerical simulation results are presented for the proposed controller. Concluding remarks are provided in Section VI.

\section{MiCRO ACTUATOR DYNAMICS}

The dynamic model of the MEA can be depicted as a spring-mass-damper assembly as shown in Fig. 1. A series resistor, $r$, is connected to the device to optimize the speedenergy characteristics [3]. The series resistor has an enormous effect on the total power dissipation during a switching transient. From Fig. 1, the dynamic model of the micro actuator can be written as [8]

$$
\begin{gathered}
m \ddot{x}=-b \dot{x}-k\left(x-x_{o}\right)-\frac{q^{2}}{2 \varepsilon A} \\
\dot{q}=\frac{1}{r}\left(v_{s}-\frac{q x}{\varepsilon A}\right)
\end{gathered}
$$

where $x(t), \dot{x}(t), \ddot{x}(t) \in \mathbb{R}$ are the displacement, velocity, and acceleration of the movable plate, respectively, $m \in \mathbb{R}^{+}$is the mass of the movable plate, $k \in \mathbb{R}^{+}$and $b \in \mathbb{R}^{+}$are the spring and damping constants, respectively, $x_{o} \in \mathbb{R}^{+}$denotes the maximum gap between the movable and fixed plates and also called the zero voltage gap, $q(t), \dot{q}(t) \in \mathbb{R}^{+}$represent the charge and current of the micro actuator, respectively, $A \in \mathbb{R}^{+}$is the plate area, and $\varepsilon \in \mathbb{R}^{+}$is the permittivity in the gap. In (2), $r \in \mathbb{R}^{+}$represents the series resistor, and $v_{S}(t) \in \mathbb{R}$ denotes the control voltage input.

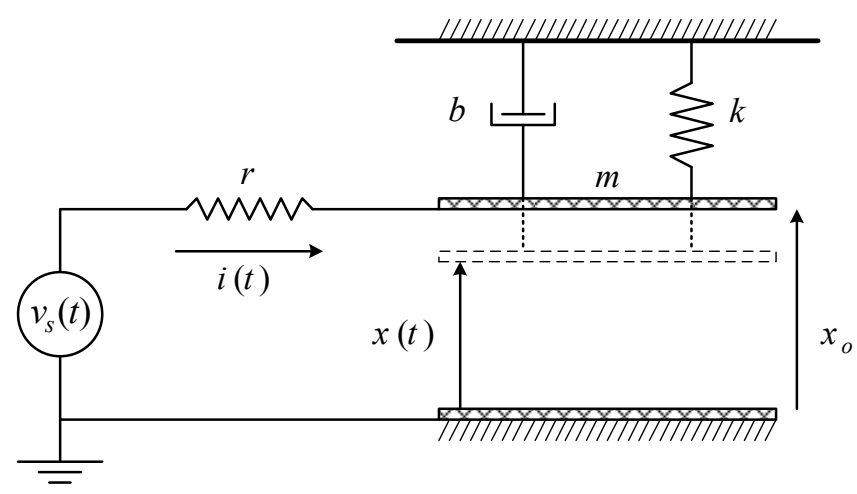

Fig. 1. Micro electrostatic actuator model. The top plate of the micro device is free to move and the bottom one is held fixed

Remark 1: The fixed (bottom) plate of the MEA is coated with an insulating material, with a thickness of $\delta_{o} \in \mathbb{R}^{+}$, to protect it from a short-circuit condition. The existence of the series resistor, $r$, in the circuit is also used as well to protect the device from the occurrence of a short-circuit condition. In addition, the displacement of the MEA's movable plate, $x(t)$, satisfies the condition $\delta_{o} \leq x \leq x_{o}$

To simplify the system analysis and control design, the MEA's dynamics in (1) and (2) is transformed into normalized coordinates as follows

$$
\begin{gathered}
\ddot{X}=-2 \xi \dot{X}-X+1-\frac{Q^{2}}{3} \\
\dot{Q}=-\frac{1}{\alpha} Q X+\frac{2}{3 \alpha} V
\end{gathered}
$$

where $X=\frac{x}{x_{o}}$ is the normalized displacement of movable plate, $Q=\frac{q}{q_{p}}$ is the normalized MEA charge, $V=\frac{v_{s}}{v_{p}}$ is the normalized control voltage, $q_{p}=\frac{3}{2} C_{o} v_{p}$ is the pull-in charge, and $v_{p}=\omega \sqrt{\frac{8 m x_{o}^{2}}{27 C_{o}}}$ is the pull-in control voltage. $C_{o}=\frac{\varepsilon A}{x_{o}}$ is the capacitance at rest, $\omega=\sqrt{\frac{k}{m}}$ is the undamped natural frequency, $\xi=\frac{b}{2 \sqrt{m k}}$ is the damping ratio, and $\alpha=r \omega C_{o}$ is a scaling constant. The normalized dynamics described in (3) and (4) are with respect to normalized time $T$ which is equal to $\omega t$ where $t$ is the time in actual dynamics. 


\section{NONLINAER CONTROLLER FORMULATION}

The subsequent controller development utilizes the measurements of the normalized MEA's movable plate displacement, $X(T)$, and internal charge, $Q(T)$. The main control objective is to design a control law for the normalized control voltage input, $V(T)$, introduced in (4), to force the movable plate's displacement, $X(T)$, to track a desired timevarying trajectory, denoted by $X_{d}(T) \in \mathbb{R}^{+}$, such that

$$
X(T) \rightarrow X_{d}(T) \text { as } T \rightarrow \infty \text {. }
$$

Assumption 1: The subsequent controller development requires that a desired time-varying trajectory is selected such that $X_{d}(T), \dot{X}_{d}(T)$, and $\ddot{X}_{d}(T)$ are bounded.

It is also required that the device charge, $Q(T)$, tracks an auxiliary signal (desired charge), $Q_{d}(T) \in \mathbb{R}$, such that

$$
Q(T) \rightarrow Q_{d}(T) \quad \text { as } \quad T \rightarrow \infty .
$$

To facilitate the control design, the following error signals, $e(T) \in \mathbb{R}$ and $\eta(T) \in \mathbb{R}$, are defined

$$
\begin{aligned}
& e \triangleq X-X_{d} \\
& \eta \triangleq Q_{d}-Q .
\end{aligned}
$$

From the definitions in (7) and (8), it is clear that if $e(T), \eta(T) \rightarrow 0 \quad$ as $T \rightarrow \infty \quad$, then $X(T) \rightarrow X_{d}(T)$ and $Q(T) \rightarrow Q_{d}(T)$ as $T \rightarrow \infty$; thus, meeting the control objectives in (5) and (6). By taking the second time derivative of $e(T)$ in (7), the following expression may be obtained

$$
\ddot{e}=-2 \xi \dot{X}-X+1-\frac{Q^{2}}{3}-\ddot{X}_{d}
$$

where (3) was utilized.

Remark 2: The velocity of the MEA's movable plate, $\dot{X}(T)$, is difficult to be measured. Hence, filtered signals are designed in Section IV to facilitate the evaluation of the velocity required in the control development.

To facilitate the control algorithm development, the term $\frac{Q_{d}^{2}}{3}$ is added to and subtracted from (9) and definition in (7) is utilized. Hence, equation (9) may be rewritten as

$$
\ddot{e}=-2 \xi \dot{X}-X+1-\frac{Q_{d}^{2}}{3}+\frac{\left(Q_{d}+Q\right)}{3} \eta-\ddot{X}_{d} .
$$

To achieve the control objectives, the auxiliary signal, $Q_{d}(T)$, is designed as

$$
Q_{d}=\sqrt{3\left(u_{F B}+\gamma\right)}
$$

where $u_{F B}(T) \in \mathbb{R}$ is the feedback control signal and $\gamma(T) \in \mathbb{R}^{+}$is defined as

$$
\gamma \triangleq 1-X_{d}-2 \xi \dot{X}_{d}-\ddot{X}_{d}
$$

Remark 3: It can be proved that the constraint $u_{F B}>-\gamma$ is satisfied for (11). In addition, $\gamma(T)$ is bounded (see Assumption 1 and (12)).

After substituting (11) with (12) in (10), the second time derivative of $e(T)$ becomes

$$
\ddot{e}=-2 \xi \dot{e}-e-u_{F B}+\frac{\left(Q_{d}+Q\right)}{3} \eta .
$$

Thus, the feedback control signal, $u_{F B}(T)$, is designed as

$$
u_{F B}=\frac{\gamma}{2}(\tanh (\lambda e)+\tanh (\lambda \dot{e}))
$$

where $\tanh (\cdot)$ is the hyperbolic tangent function and $\lambda \in \mathbb{R}^{+}$ is a control gain. The closed-loop error dynamics in (13) can be rewritten as

$$
\ddot{e}=-g(e)-h(\dot{e})+\frac{\left(Q_{d}+Q\right)}{3} \eta
$$

where (14) was utilized and the terms $g(e)$ and $h(\dot{e})$ are defined as

$$
\begin{gathered}
g(e) \triangleq e+\frac{\gamma}{2} \tanh (\lambda e) \\
h(\dot{e}) \triangleq 2 \xi \dot{e}+\frac{\gamma}{2} \tanh (\lambda \dot{e}) .
\end{gathered}
$$

Property 1: It is clear from the definitions in (16) and (17) that $g(0)=h(0)=0$ and $\tau g(\tau)>0$ as well as $\tau h(\tau)>0$ are satisfied for all $\tau$.

By taking the first time derivative of the error signal in (8), the following closed-loop error dynamics can be obtained for $\eta(T)$ as

$$
\dot{\eta}=\dot{Q}_{d}+\frac{1}{\alpha} Q X-\frac{2}{3 \alpha} V
$$

where (4) was utilized. Based on the subsequent stability analysis, the control voltage input $V(T)$ is designed as

$$
V=\frac{3 \alpha}{2}\left[\dot{Q}_{d}+\frac{1}{\alpha} Q X+k_{s} \eta+\frac{1}{3}\left(Q_{d}+Q\right) \dot{e}\right]
$$

where $k_{s} \in \mathbb{R}^{+}$is a control gain and $\dot{Q}_{d}$ is computed to be

$$
\dot{Q}_{d}=\frac{3 \gamma \lambda}{4 Q_{d}}\left(\operatorname{sech}^{2}(\lambda e) \dot{e}+\operatorname{sech}^{2}(\lambda \dot{e}) \ddot{e}\right) .
$$

Thus, the closed-error dynamics of $\eta(T)$ becomes

$$
\dot{\eta}=-k_{s} \eta-\frac{1}{3}\left(Q_{d}+Q\right) \dot{e}
$$


Remark 4: The control voltage input, $V(T)$, designed in (19) requires the measurements of the MEA's normalized charge $Q(T)=\frac{q}{q_{p}}$. The device actual charge $q(t)$ can be measured indirectly utilizing the measurement the voltage drop $v_{r}(t)$ across the series resistor, $r$, and actual MEA's movable plate displacement $x(t)$ such that $q=\frac{\varepsilon A}{x}\left(v_{s}-v_{r}\right)$ where the voltage drop across the device is $v_{\text {device }}=v_{s}-v_{r}=\frac{q x}{\varepsilon A}$.

Theorem 1: The controller, given in (11) and (19), ensures that $e(T), \eta(T) \rightarrow 0$ as $T \rightarrow \infty$ and all closedloop signals are bounded. Hence, $X(T) \rightarrow X_{d}(T) \quad$ and $\quad Q(T) \rightarrow Q_{d}(T) \quad$ as $T \rightarrow \infty$.

Proof: By utilizing the Lyapunov function $V_{1}=\frac{1}{2} \dot{e}^{2}+\int_{0}^{e} g(\sigma) \sigma+\frac{1}{2} \eta^{2}$, it can be shown that the proposed controller, given in (11) and (19), provides a global asymptotic result. Details have been omitted due to limited space.

\section{FILTER DESIGN}

The error signal $\dot{e}(T)$, utilized in the control design in (14) and (19), is not available due to the difficulty in obtaining the measurement of the MEA's movable plate's velocity, $\dot{X}(T)$, hence; filter-based approach is adopted to facilitate the computation of the error signal $\dot{e}(T)$. The following filters are designed

$$
\begin{gathered}
e_{f_{1}}=e_{f_{2}}+\dot{e}+e \\
\dot{e}_{f_{2}}=-e_{f_{2}}-k_{f} e_{f_{1}}+e .
\end{gathered}
$$

By substituting (22) into (23), the following expression can be obtained

$$
\dot{e}_{f_{2}}=\dot{e}_{f_{3}}-k_{f} \dot{e}
$$

where

$$
\dot{e}_{f_{3}} \triangleq-\left(k_{f}+1\right) e_{f_{2}}-\left(k_{f}-1\right) e .
$$

It is clear from (24) that the following expression can be obtained

$$
e_{f_{2}}=e_{f_{3}}-k_{f} e .
$$

The expression in (25) can be rewritten as

$$
\dot{e}_{f_{3}}=-\left(k_{f}+1\right) e_{f_{3}}+\left(k_{f}^{2}+1\right) e
$$

where (26) was utilized. After taking the first time derivative of (22) and utilizing (9), the following expression is obtained

$$
\dot{e}_{f_{1}}=\dot{e}_{f_{2}}-2 \xi \dot{X}-X+1-\frac{Q^{2}}{3}-\ddot{X}_{d}+\dot{e} .
$$

The expression in (28) can be rewritten as

$$
\dot{e}_{f_{1}}=\dot{e}_{f_{4}}-2 \xi \dot{X}
$$

where

$$
\dot{e}_{f_{4}} \triangleq-\left(k_{f}-1\right) e_{f_{1}}-2 e_{f_{2}}-X+1-\frac{Q^{2}}{3}-\ddot{X}_{d}
$$

and (22) as well as (23) were utilized. It is clear from (29) that the following expression can be obtained

$$
e_{f_{1}}=e_{f_{4}}-2 \xi X .
$$

The definition in (30) can be rewritten as

$$
\begin{array}{r}
\dot{e}_{f_{4}} \triangleq-\left(k_{f}-1\right) e_{f_{4}}-2 e_{f_{2}}+\left[\left(k_{f}-1\right) 2 \xi-1\right] X \\
+1-\frac{Q^{2}}{3}-\ddot{X}_{d}
\end{array}
$$

where (31) was utilized.

Remark 5: It is clear that $e_{f_{1}}(T)$ can be computed from (31) utilizing $e_{f_{4}}(T)$ that is computed from (32). Note that the expression in (32) utilizes $e_{f_{2}}(T)$. The signal $e_{f_{2}}(T)$ can be computed from (26) utilizing $e_{f_{3}}(T)$ that is computed from (27). Hence, the error signal $\dot{e}(T)$ can be computed from (22) knowing that $e_{f_{1}}(T)$ and $e_{f_{2}}(T)$ are computable. Based on the previous fact, the velocity of the MEA's movable plate, $\dot{X}(T)$, can be evaluated utilizing the definition in (7).

\section{NUMERICAL SIMULATION}

Numerical simulations are performed to demonstrate the effectiveness of the proposed controller introduced in (11) and (19). The system model parameter values [3] were chosen as shown in Table I.

TABLE I

NUMERICAL MODEL PARAMETER VALUES

\begin{tabular}{|c|c|c|}
\hline \hline Parameter & Value & Unit \\
\hline \hline$m$ & $3.29 \times 10^{-10}$ & {$[\mathrm{~kg}]$} \\
\hline$k$ & 476 & {$[\mathrm{~N} / \mathrm{m}]$} \\
\hline$b$ & $8 \times 10^{-4}$ & {$[\mathrm{~N} . \mathrm{s} / \mathrm{m}]$} \\
\hline$\varepsilon$ & $8.85 \times 10^{-12}$ & {$[\mathrm{~F} / \mathrm{m}]$} \\
\hline$A$ & $6.2 \times 10^{-8}$ & {$\left[\mathrm{~m}^{2}\right]$} \\
\hline$r$ & $0.91 \times 10^{+6}$ & {$[\Omega]$} \\
\hline$x_{o}$ & $2 \times 10^{-6}$ & {$[\mathrm{~m}]$} \\
\hline$X(0)$ & 0.2 & - \\
\hline
\end{tabular}


The control gains $k_{s}, k_{f}$, and $\lambda$ were tuned and selected to be 200, 200, and 50, respectively, so that the best performance was achieved.

In Fig. 2., the response of the MEA's actual normalized displacement is introduced for a desired time-varying displacement (i.e., $\left.X_{d}=0.4 \sin (0.1 \pi T)+0.45\right)$. Note that the normalized frequency selected for the desired time-varying displacement is equal to $0.05 \mathrm{~Hz}$ and this implies that the actual frequency required to be tracked by the MEA is about $f \omega \cong 60 \mathrm{kHz}$ where $\omega$ was defined at the end of Section II. Figures 3, 4, 5 and 6 present the normalized displacement tracking error, normalized velocity, normalized control voltage input, normalized MEA's internal charge, respectively. It was observed that the MEA was successfully tracked.

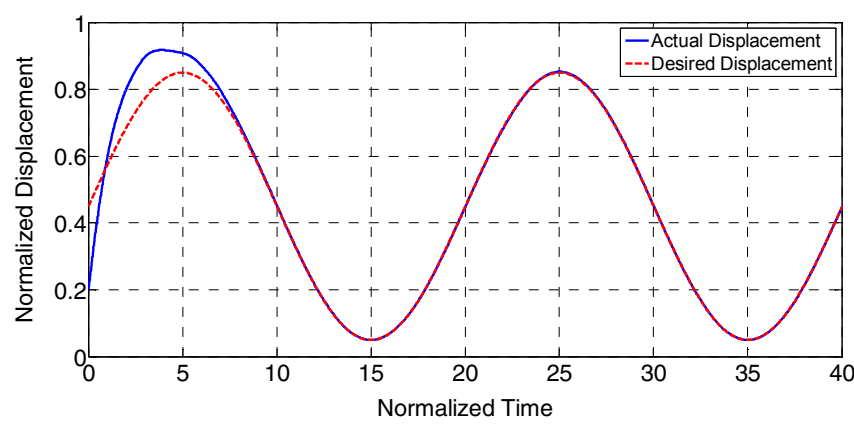

Fig. 2. Simulated MEA's normalized actual displacement vs. desired one.

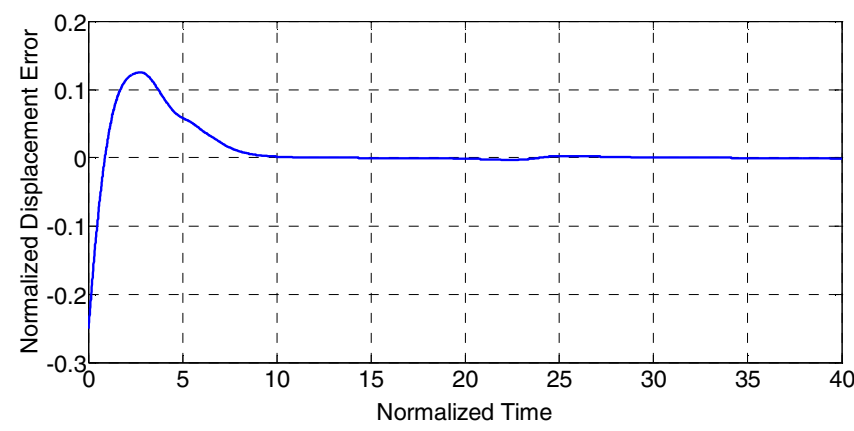

Fig. 3. Simulated MEA's normalized displacement tracking error.

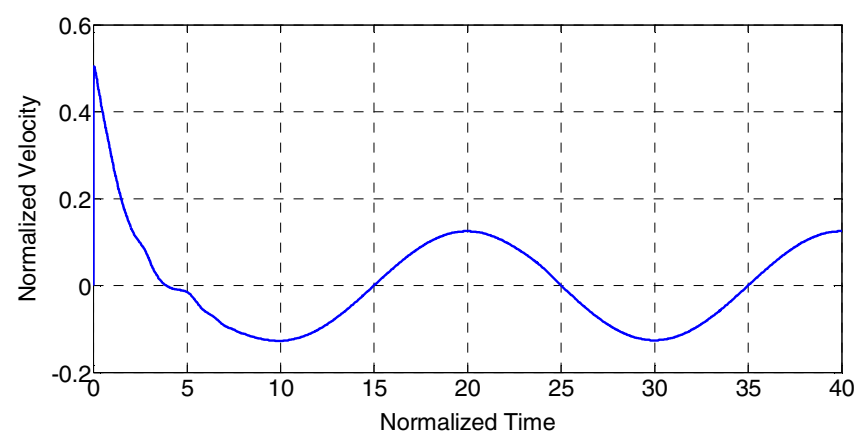

Fig. 4. Simulated MEA's normalized velocity.

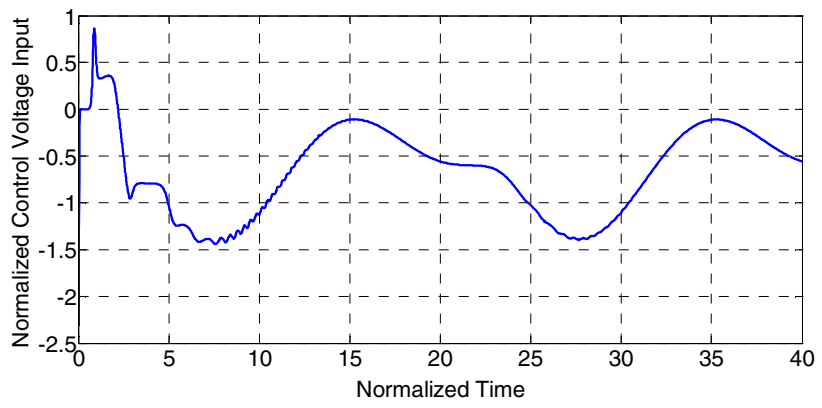

Fig. 5. Simulated MEA's normalized control voltage input.

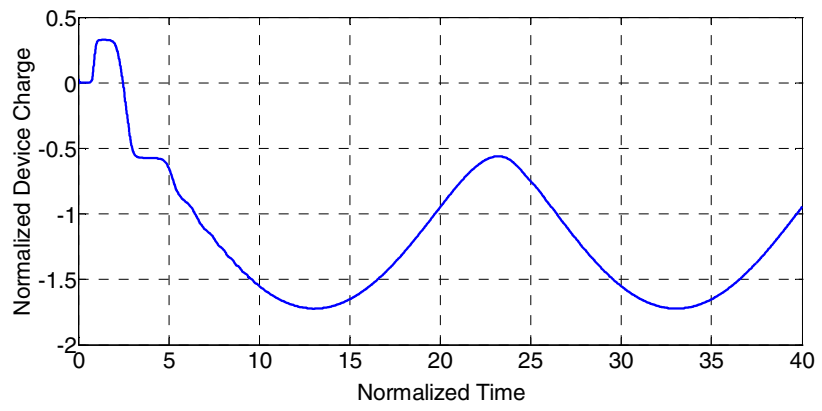

Fig. 6. Simulated MEA's normalized device charge.

From figures 5 and 6 , it is noted that the actual applied control voltage input $v_{s}=V \cdot v_{p}$ and the actual internal charge of the device $q=Q \cdot q_{p}$ exceed the negative of the pull-in value and that was needed to track the desired timevarying displacement as was showed in [12].

To demonstrate the performance of the nonlinear controller proposed in (11) and (19), a standard PID controller was utilized for comparison purposes. The following measures were computed to quantify the performance of each controller,

$$
\begin{aligned}
& M_{e} \triangleq \int_{0}^{T}|e(\tau)|^{2} d \tau \\
& M_{u} \triangleq \int_{0}^{T}|V(\tau)|^{2} d \tau
\end{aligned}
$$

where $M_{e}(T)$ and $M_{u}(T)$ are the measure of error magnitude and the measure of energy expanded by the controller over the period of system operation $(T=120)$. The desired time-varying displacement was chosen to be

$$
\begin{aligned}
X_{d}= & 0.1[\sin (0.1 t)+\sin (0.2 t)+\sin (0.3 t)+\sin (0.4 t)] \\
& +0.5
\end{aligned}
$$

The standard PID controller was defined as

$$
V \triangleq K_{p} e+K_{I} \int_{0}^{T} e(\tau) d \tau+K_{D} \frac{d e}{d T}
$$

where the PID controller gains were tuned and chosen to be $K_{p}=8, K_{I}=2$, and $K_{D}=1$ and for the proposed nonlinear controller were selected to be $K_{s}=50, K_{f}=200$, and $\lambda=25$ and $X(0)=0.9$ in order to demonstrate the best performance 
to the MEA dynamic system. Figures 7 and 8 present the MEA's normalized displacement with respect to the desired time-varying displacement and normalized displacement tracking error, respectively, when the proposed nonlinear controller is compared with a standard PID controller.

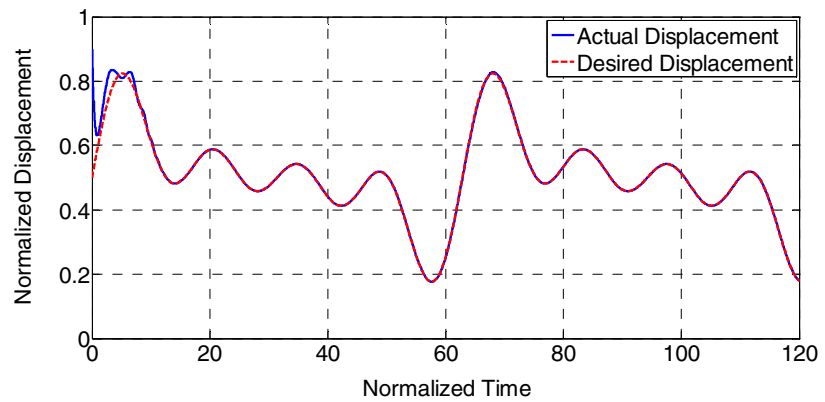

Fig. 7. Simulated MEA's normalized actual displacement for the nonlinear controller versus the desired displacement.

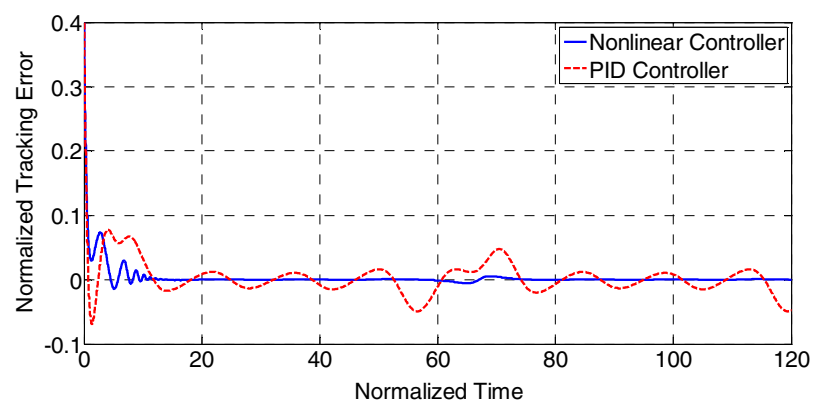

Fig. 8. Simulated MEA's normalized displacement tracking error for both the nonlinear controller versus the standard PID controller.

Table II shows a performance comparison for the proposed nonlinear controller and the standard PID controller. It can clearly be seen from Table II that improved tracking performance is achieved by using the filter-based nonlinear controller by $53.7 \%$ in the tracking error and by $29.5 \%$ in the control effort.

TABLE II

COMPARISON OF MEASURES FOR DIFFERENT CONTROLLERS

\begin{tabular}{|c|c|c|}
\cline { 2 - 3 } \multicolumn{1}{c|}{} & $M_{e}(T)$ & $M_{u}(T)$ \\
\hline \hline Nonlinear Controller & 0.044 & 101 \\
\hline PID Controller & 0.095 & 143.2 \\
\hline \hline
\end{tabular}

From figures 6 and 7 as well as Table II, it is clear that the nonlinear controller demonstrated a satisfactory performance when compared with a standard PID controller.

\section{REFERENCES}

[1] S. Bermejo, and L. Castaner, "Dynamics of MEMS electrostatic driving using a photovoltaic source," Sensors and Actuators A: Physical, vol. 121, no. 1, pp. 237-242, May 2005.

[2] L. Castaner, J. Pons, R. Nadal-Guardia, and A. Rodriguez, "Analysis of the extended operation range of electrostatic actuators by current-pulse drive," Sensors and Actuators A: Physical, vol. 90, no. 3, pp. 181-190, May 2001.

[3] L. Castaner, and S. Senturia, "Speed-energy optimization of electrostatic actuators based on pull-in," IEEE Journal of
Microelectromechanical Systems, vol. 8, no. 3, pp. 290-298, September 1999.

[4] A. Fargas-Marques, J. Casals-Terre, and A. Shkel, "Resonant pull-in condition in parallel-plate electrostatic actuators," IEEE Journal of Microelectromechanical Systems, vol. 16, no. 5, pp. 1044-1053, October 2007.

[5] W. Li, and P. Liu, "Robust adaptive tracking control of uncertain electrostatic micro-actuators with H-infinity performance," Mechatronics, vol. 19, no. 5, pp. 591-597, 2009

[6] M. Lu, and G. Fedder, "Closed-loop control of a parallel-plate microactuator beyond the pull-in limit," in Proceedings of the SolidState Sensor, Actuator and Microsystems Workshop, Hilton Head Island, South Carolina, June 2002, pp. 255-258.

[7] M. Lu, and G. Fedder, "Position control of parallel plate microactuators for probe-based data storage," IEEE Journal of Microelectromechanical Systems, vol. 13, no. 5, pp. 759-769, October 2004.

[8] D. Maithripala, J. Berg, and W. Dayawansa, "Control of an electrostatic microelectromechanical system using static and dynamic output feedback," ASME Journal of Dynamic Systems, Measurement, and Control, vol. 127, no. 3, pp. 443-450, September 2005.

[9] J. Pons-Nin, A. Rodriguez, and L Castaner, "Voltage and pull-in time in current drive of electrostatic actuators," IEEE Journal of Microelectromechanical Systems, vol. 11, no. 3, pp. 196-205, June 2002.

[10] D.-Y. Qiao, W.-Z. Yuan, and X.-Y. Li, "A two-beam method for extending the working range of electrostatic parallel-plate microactuators," Journal of Electrostatics, vol. 65, no. 4, pp. 256-262, April 2007.

[11] L. Rocha, E. Cretu, and R. Wolffenbuttel, "Using dynamic voltage drive in a parallel-plate electrostatic actuator for full-gap travel range and positioning," IEEE Journal of Microelectromechanical Systems, vol. 15, no. 1, pp. 69-83, February 2006.

[12] J. Seeger, and B. Boser, "Dynamics and control of parallel-plate actuators beyond the electrostatic instability," in Proceedings of the 10th International Conference on Solid-State Sensors and Actuators, Sendai, Japan, June 1999, pp. 474-477.

[13] J. Seeger, and B. Boser, "Charge control of parallel-plate, electrostatic actuators and the tip-in instability," IEEE Journal of Microelectromechanical Systems, vol. 12, no. 5, pp. 656-671, October 2003.

[14] J. Seeger, and S. Crary, "Stabilization of electrostatically actuated mechanical devices," in Proceedings of the International Conference on Solid-State Sensors and Actuators, Chicago, June 1997, pp. 1133-1136.

[15] K. Tee, S. Ge, and F. Tay, "Adaptive Control of Electrostatic Microactuators with Bidirectional Drive," IEEE Transactions on Control System Technology, vol. 17, no. 2, pp. 340-352, March 2009.

[16] M. Vagia, G. Nikolakopoulos, and A. Tzes, "Design of a robust PIDcontrol switching scheme for an electrostatic micro-actuator," Control Engineering Practice, vol. 16, no. 11, pp. 1321-1328, November 2008.

[17] J. Zara, and S. Smith, "Optical scanner using a MEMS actuator," Sensors and Actuators A: Physical, vol. 102, no. 1-2, pp. 176-184, December 2002.

[18] G. Zhu, J. Levine, and L. Praly, "Improving the performance of an electrostatically actuated MEMS by nonlinear control: some advances and comparisons," in Proceedings of the 44th IEEE Conference on Decision and Control, and the European Control Conference, Seville, Spain, pp. 7534-7539, Dec 2005.

[19] G. Zhu, J. Levine, L. Praly, and Y.-A. Peter, "Flatness-based control of electrostatically actuated MEMS with application to adaptive optics: a simulation study," IEEE Journal of Microelectromechanical Systems, vol. 15 , no. 5 , pp. 1165-1174, Oct 2006.

[20] G. Zhu, M. Packirisamy, M. Hosseini, and Y.-A. Peter, "Modelling and control of an electrostatically actuated torsional micromirror," Journal of Micromechanics and Microengineering, vol. 16, no. 10, pp. 2044-2052, Oct 2006.

[21] G. Zhu and L. Saydy, "Robust output feedback control of an electrostatic micro-actuator," in Proceedings of the American Control Conference, New York City, NY, pp. 3192-3197, July 2007.

[22] G. Zhu, J. Penet, and L. Saydy, "Modeling and control of electrostatically actuated MEMS in the presence of parasitics and parametric uncertainties," ASME Journal of Dynamic Systems, Measurement, and Control, vol. 129, no. 6, pp. 786-794, Nov 2007. 